\title{
EDITORIAL
}

\section{Classification of depressive illness: a re-evaluation}

The debate as to whether depression is unimodally or bimodally distributed has rotated around studies whose variables have included precipitating events, events consequent upon the illness, personality traits, and inessential symptoms in addition to uniquely defining characteristics. The confusion caused by failing to distinguish between differentiae and descriptive symptoms was discussed at some length by Foulds (1962) and has been emphasized by Gruenberg (1969).

'These specifications' [as to the way in which each disorder differs from other disorders] 'should be confined to the crucial differences if they are to avoid introducing descriptions of additional characteristics which ... do not enter into the reasons for recognizing that this particular form of mental disorder differs from other forms'.

A study by Kiloh and Garside (1963) may serve as an example: 'The first factor', they said, 'is a general factor and thus the factor loading indicates the extent to which each feature is related to all the features as a whole, that is to depressive illness as defined by the sum of the 35 features' (our italics). We do not consider this a legitimate way in which to define depression. It is a description of depressed persons who had already been clinically selected as such. The first factor, therefore, shows those features which are common to the two groups described by their second factor; but these features do not necessarily differentiate between depressives and non-depressives. Indeed, when one examines them, one would scarcely expect them to do so. Ten of the 35 symptoms, personality traits or precipitating events had loadings of .24 or more on factor 1 . These were: (1) failure of concentration; (2) agitation; (3) suicidal ideas; (4) reactive depression; (5) hysterical features; (6) self-reproach -guilt; (7) hysterical features-immaturity; (8) irritability; (9) inadequacy; and (10) subjective anxiety. These polymorphous features, whether singly or in combination, are not sufficient to differentiate between depression and not-depression as a diagnosis. Few could even be considered as necessary for depression. Precipitating or consequent events cannot constitute necessary and sufficient conditions for the identification of depression. In order to be able to say ' $X$ causes $D$ ' or ' $Y$ is a consequence of D', D must first have been identified. $\mathrm{X}$ and $\mathrm{Y}$ can only be descriptive of an already known condition.

Statistics can help us to decide that some features ought to be discarded; they are of little value in deciding what ought to have been included originally. As Costello (1970) has pointed out, 'Statistical procedures themselves cannot provide the answer to the question of continuity or discontinuity'; nor can they determine how many dimensions are needed to differentiate between neurotic and psychotic depression. The nature of the distributions and the number of dimensions are both dependent upon the measures selected and their importance cannot be decided solely by statistics. It would be perfectly feasible to describe men and women in such a way that summated scores on the descriptive items produced a unimodal curve. However gratifying this might be to extremists in Women's Lib, an individual's position on the continuum would not, as again Costello (op. cit.) has noted, be of surpassing interest to an obstetrician.

There are three necessary steps before distinguishing between psychotic and neurotic depression (or delusions of Contrition and state of Depression, as we would prefer). We must distinguish between: (1) depression and not-depression; (2) psychopathological and not-psychopathological depression; and (3) psychosis and neurosis. Before attempting to make these distinctions, it is necessary to describe the proposed hierarchy of classes of personal illness (Foulds, 1965, 1971; Foulds and Bedford, 1975).

It has been suggested that it may prove useful to think of personal illness as falling into four classes, 
which involve decreasing degrees of disruption of the normal, or premorbid, personality and of the ability to enter into and maintain mutual personal relationships.

The most severe class has been called Delusions of Disintegration (class 4 ). This would be expected to be made up largely of schizophrenics. Groups, if any, within this class have not yet been decided upon. The next class has been termed Integrated Delusions (class 3), which is composed of delusions of Contrition, of Grandeur and of Persecution. These groups might be expected to be made up mainly of psychotic depressive, manic, and paranoiac patients respectively. The next class is that of Neurotic Symptoms (class 2). Here the groups are Conversion, Dissociative, Phobic, Compulsive, and Ruminative symptoms. The last class is that of Dysthymic States (class 1) and the groups are states of Anxiety, of Depression, and of Elation. We have argued that states of Anxiety and of Depression should not be included in the Neurotic Class (Foulds and Bedford, op. cit.). Those with phobic symptoms have been found almost invariably $(95 \%)$ to have anxiety states; whereas the converse does not hold. Only $44 \%$ of those who fall into the Anxiety state group also fall into the Phobic group. The relationship is, therefore, an inclusive, non-reflexive, one.

It has been claimed not only that the higher class takes precedence over the lower, but that the higher class includes the lower. Thus, all persons within class 4 have symptoms pertaining to classes 3,2 , and 1 ; all those with class 3 symptoms have some symptoms of classes 2 and 1 ; all those with class 2 symptoms have some symptoms of class 1 . On the other hand, not all with class 3 symptoms have class 4 symptoms; not all with class 2 symptoms have symptoms of classes 3 and 4 ; not all those with class 1 symptoms have symptoms of classes 2, 3, and 4. It is suggested, therefore, that the hierarchy consists of a series of inclusive, non-reflexive, class relationships.

Essentially the same point has been made by Gruenberg (op. cit.).

[APA classification] 'has a simple logical structure in which the manifestations of each condition can include the manifestations of the subsequent conditions, but the later conditions do not include the manifestations of the conditions listed earlier'. He goes on to say that 'it could mean that the genius of the psychiatric profession has over the years discovered a latent logic inherent in mental disorders. But I am more inclined to think that we have simply imposed on the clinical phenomena which come to our attention a classification scheme in which we give logical priority to conditions with the most varied manifestations and recognize that some forms of mental disorder are less heterogeneous in their manifestations'.

We agree with this view, but regret the lack of systematic investigations designed to evaluate the utility of such a logical structure. Wing et al. (1974) also consider that 'clinical diagnosis is hierarchical' and more fragmentary indications of a hierarchical notion can be found scattered throughout the psychiatric literature, beginning perhaps with Ernst von Feuchtersleben in 1845. 'Every psychosis', he states, 'is, at the same time, a neurosis; because without the intervention of nervous action, no change of the psychical action becomes manifest, but every neurosis is not a psychosis, of which convulsions and pain afford sufficient examples'. Certainly he was using the terms psychosis and neurosis differently from the current usage-rather more akin to the person and the organism; but he was undoubtedly putting forward the notion of an inclusive, non-reflexive, relationship. One of the most eminent psychiatrists to so do was Eugene Bleuler (1950).

'All the phenomena of manic-depressive psychosis may also appear in our disease; the only decisive factor is the presence or absence of schizophrenic symptoms. Therefore, neither a state of manic exaltation nor a melancholic depression, nor the alternation of both states has any significance for the diagnosis. Only after careful observation has revealed no schizophrenic features may we conclude that we are dealing with a manic-depressive psychosis'.

Those who wish to pursue our unoriginality further will find hints of such relationships in Berrington et al. (1956), Clark and Mallett (1963), Chapman (1966), Gittleson (1966), Maxwell (1972, 1973), and Priest et al. (1973) among many others.

We now wish to return to the three necessary steps before distinguishing between psychotic and neurotic depression. 
DEPRESSION V. NOT-DEPRESSION A person will generally be considered to be depressed if either he claims to have experienced for at least some days or weeks an appreciable lowering of mood and/or observers infer from his tone of voice, facial expression, content of speech, etc. that he is experiencing an appreciable lowering of mood. Without this no person could reasonably be described as depressed; with it, everyone would be so described. The presence of other symptoms higher in the hierarchy might, however, require that the diagnosis be something other than Depression.

PSYCHOPATHOLOGICAL v. NOT-PSYCHOPATHOLOGICAL DEPRESSION Freud (1917) made a distinction between psychotic depression and not-psychopathological depression which is still widely accepted.

'The distinguishing mental features of melancholia are a profoundly painful dejection, cessation of interest in the outside world, loss of the capacity to love, inhibition of all activity, and a lowering of the self-regarding feelings to a degree that finds utterance in self-reproaches and self-revilings, and culminates in a delusional expectation of punishment. The picture becomes a little more intelligible when we consider that, with one exception, the same traits are met with in mourning. The disturbance of self-regard is absent in mourning; but otherwise the features are the same ... although mourning involves grave departures from the normal attitude to life, it never occurs to us to regard it as a pathological condition and to refer it to medical treatment'.

Laxer (1964) takes a similar stand when discussing neurotic depression. He believes that a combination of very low mood (Freud's 'profoundly painful dejection') and high self-blame (Freud's 'self-revilings') is a necessary, if not sufficient, condition for the syndrome of neurotic depression. Clearly, outside a hierarchy model, this combination is not a sufficient condition, since it occurs, for example, in schizophrenia, in psychotic depression, and in obsessional neurosis; but it is sufficient to distinguish between psychopathological and not-psychopathological depression. Within the hierarchy model, it is a sufficient condition for a state of Depression in the absence of symptoms belonging to classes higher in the hierarchy - that is, neurotic or psychotic symptoms.

PSYCHOSIS v. NEUROSIS The conditions required for this differentiation, as for others, must be found in one class, but not in the other. Wing et al. (op. cit.) give, as the chief symptoms of Depressive Psychoses, depressive mood and depressive delusions or hallucinations and, as the chief symptoms of Neurotic Depression, depressive mood and anxiety. Without the hierarchy, one would be forced to conclude that the respective differentiae were depressive delusions and anxiety, since depressive mood is common to both groups. With the hierarchy, the respective differentiae are depressive delusions on the one hand and, on the other, depressive mood in the absence of depressive delusions (or any other symptoms higher in the hierarchy).

We would, therefore, define a state of Depression as a profoundly painful dejection together with high self-blame (thus excluding 'normal' depression) in the absence of neurotic symptoms or delusions. Psychotic Depression we are equating with delusions of Contrition, in the absence of delusions of Disintegration (Autochthonous, passivity, influence). This is in accord with an earlier finding (Foulds, 1960), which was supported by Beck (1967), who concluded 'that there are no specific signs or symptoms, aside from delusions, that distinguish psychotic from neurotic depressives'.

We have administered the self-report Delusions-Symptoms-States Inventory (DSSI), which is concerned with recent state, to over 550 patients and 260 normal subjects. There are 12 sets of seven items each, which conform exactly to the hierarchy of classes of personal illness and their constituent groups outlined above.

Items answered 'False' always score 0 . In nearly all instances in the eight sets comprising the Dysthymic States and Neurotic Symptoms classes, if the person affirmed a question, he has additionally to indicate the extent to which it upsets him-for example, a bit, a lot, or almost unbearably. Occasionally, particularly for the state of Elation, he indicates the frequency of occurrence-for example, seldom, often, or nearly always. Scores of 1,2 , or 3 are given in the direction of increasing distress or frequency. A person affirming any delusional item has to indicate the degree of his conviction-for example, not very sure, fairly sure, or certain. These are likewise scored 1, 2, or 3. 
Those who score 4 or more on any set (which requires the endorsement of at least two items) are allocated to that group.

From the 550 patients we have compared the 68 who fell into the delusions of Contrition group (but no other delusional group) with the 47 who fell into the state of Depression group only (with the exception of state of Anxiety with which there is the expected considerable overlap. Even here only those who scored higher on Depression than on Anxiety were included).

Of the 68 who scored 4 plus on delusions of Contrition (dC), $66(97 \%)$ scored 4 plus on state of Depression. The remaining two scored 4 plus on state of Anxiety. Of these $68 \mathrm{dCs}, 61(90 \%)$ fell into one or more of the Neurotic Symptoms groups (71\% into the Ruminative group; $46 \%$ Phobic; $44 \%$ Compulsive; $34 \%$ Conversion, and $32 \%$ Dissociative). The vast majority of those with an unmixed DSSI 'diagnosis' of delusions of Contrition are, therefore, Neurotic and all are Dysthymic States (invariably states of Depression and/or Anxiety, most usually both).

Unimodalists acknowledge that the ends of their distributions look very like neurotic and psychotic depression, but claim that the bulk of cases are mixed (Kendell, 1968). According to our data, those with delusions of Contrition are mixed-that is, also have a state of Depression-those with a state of Depression are not.

Nine subjects with a DSSI 'diagnosis' of state of Depression and 10 with delusions of Contrition have so far been re-tested after four weeks. Of the nine states of Depression, seven became symptomfree, one remained a state of Depression, and one additionally developed Phobic symptoms.

Nine of the 10 with delusions of Contrition fitted the hierarchy on first testing - that is, had symptoms in classes 2 and 1 and all 10 did so on retest, despite marked changes in symptomatology. One became worse by adding delusions of Disintegration. Another case remained an Integrated Psychotic, but with delusions of Grandeur replacing Contrition. As he now also came up on state of Elation, he appears to have swung from depression to mania. Four moved down the hierarchy to class 2 (one Phobic; one Ruminative; one Compulsive; one both Ruminative and Phobic); four moved down to class 1 (two states of Elation; one Depression with lesser Anxiety and one Elation with lesser Anxiety). None of the $10 \mathrm{dCs}$ had become symptom-free.

Symptoms higher up the hierarchy appear to remit before those lower in the hierarchy. It is difficult to accommodate this movement down the hierarchy within a disease-entity model. Strict adherence to this model should entail treating a patient throughout in the light of the original diagnosis. Thus, a psychotic depressive will probably be given ECT or an antidepressant drug and later will be assessed as very much improved, improved, or unimproved. Complete acceptance of a disease-entity model should entail that 'improved' refers to the symptoms of psychotic depression alone.

In the present hierarchy terms, it may be that 'very much improved' would refer to those patients who had moved down the hierarchy to class 0 (the symptom-free); 'somewhat improved' to those who had moved down to class 2 or 1 . A study designed to investigate these assumptions might be of interest.

Again, strictly according to a disease-entity model, the depressed patient who has moved down from class 3 to class 2 should be given another course of ECT or more drugs; but, according to the hierarchy model, the phobic symptoms (or whatever it was that remained) might be treated by the method the clinician thought most appropriate for that condition-for example, behaviour therapy. It might be justifiable to carry out a study designed to compare the results of working strictly within a disease-entity or a hierarchy model.

It has been argued that, even if the present view be viable, it may not logically exclude the dualist view of depression. It does. In the strict dualist view one is either a ping or a pong. We are saying that all pings are pongs, but not all pongs are pings. The dualist view allows one to be a ping, but not a pong; our view does not. Being a ping, but not a pong and being a ping and, therefore, inevitably a pong would entail that one could at one and the same time be both a pong and not a pong. The views are, therefore, logically incompatible.

Assuming, with Wing et al. (op. cit.), that $\mathrm{dC}$ virtually equals Psychotic Depression and that $\mathrm{sD}$ 
virtually equals Neurotic Depression (or state of Depression, sD), the relationship between them appears to be an inclusive, non-reflexive, one. Even those who do not accept these two equations would be unlikely to deny the salient importance in these two clinical conditions of $\mathrm{dC}$ and $\mathrm{sD}$. It is perhaps improbable, therefore, that any classification, which was similarly confined to the universe of symptoms and signs, would produce radically different results.

We conclude, therefore-subject, of course, to replication by disinterested or antagonistic workers -that (1) those with a preponderance of delusions of Contrition over delusions of Grandeur or of Persecution, may, in the absence of delusions of Disintegration, be regarded as virtually synonymous with Psychotic Depressives; (2) dCs (or Psychotic Depressives) almost invariably suffer from Neurotic Symptoms (with Ruminations the commonest); (3) all dCs (or Psychotic Depressives) suffer from either a state of Anxiety or of Depression and usually both; (4) a state of Depression, as a diagnosis, can only be identified in the absence of Neurotic Symptoms (Phobic, Hysterical, or Obsessional) and of Delusions.

\section{G. A. FOULDS AND A. BEDFORD}

\section{REFERENCES}

Beck, A. T. (1967). Depression. Hoeber: New York.

Berrington, W. P., Liddell, D. W., and Foulds, G. A. (1956). A re-evaluation of the fugue. Journal of Mental Science, 102, 280-286.

Bleuler, E. (1950). Dementia Praecox, p. 304. International Universities Press: New York.

Chapman, J. (1966). The early symptoms of schizophrenia, British Journal of Psychiatry, 112, 225-251.

Clark, J. A., and Mallett, B. L. (1963). A follow up study of schizophrenia and depression in young adults. British Journal of Psychiatry, 109, 491-499.

Costello, C. G. (ed.) (1970). Symptoms of Psychopathology. Wiley: New York.

Feuchtersleben, E. von (1845). The principles of medical, psychology, being the outlines of a course of lectures by Baron Ernst von Fcuchtersleben. The Principles of Medical Psychology. Revised and edited by B. G. Babington, 1847: Sydenham Society: London. Quoted in Three Hundred Years of Psychiatry, pp. 951-954. By R. Hunter and 1. Macalpine. Oxford University Press: London. 1963.

Foulds, G. A. (1960). Psychotic depression and age. Journal of Mental Science, 106, 1394-1397.

Foulds, G. A. (1962). A quantification of diagnostic differentiae. Journal of Mental Science, 108, 389-405.

Foulds, G. A. (1965). Personality and Personal Illness. Tavistock Publications: London.

Foulds, G. A. (1971). Personality deviance and personal symptomatology. Psychological Medicine, 1, 222-233.

Foulds, G. A., and Bedford, A. (1975). The hierarchy of classes of personal illness. Psychological Medicine. (In press.)
Freud, S. (1917). Mourning and melancholia. In Standard Edition of the Complcte Psychological Works of Signund Freud, 1957, vol. 14. Hogarth Press: London.

Gittleson, N. L. (1966). The phenomenology of obsessions in depressive psychosis. British Journal of Psychiatry, 112, 261-264.

Gruenberg, E. M. (1969). How can the new Diagnostic Manual help? International Journal of Psychiatry, 7, 368-374.

Kendell, R. E. (1968). The Classification of Depressive Illnesses. Maudsley Monographs No. 18. Oxford University Press: London.

Kiloh, L. G., and Garside, R. F. (1963). The independence of neurotic depression and endogenous depression. British Journal of Psychiatry, 109, 451-463.

Laxer, R. M. (1964). Relation of real self-rating to mood and blame and their interaction in depression. Journal of Consulting Psychology, 28, 538-546.

Maxwell, A. E. (1972). Difficulties in a dimensional description of symptomatology. British Journal of Psychiatry, 121, 19-26.

Maxwell, A. E. (1973). Psychiatric illnesses: some inferences from symptomatology. British Journal of Psychiatry, 122, 251-258.

Priest, R. G., Shariatmadari, M. E., and Tarighati, S. (1973). Affective states in schizophrenia. British Journal of Social and Clinical Psychology, 12, 283-288.

Wing, J. K., Cooper, J. E., and Sartorius, N. (1974). Measurement and Classification of Psychiatric Symptoms. Cambridge University Press: London. 